\title{
Radiatively Driven Winds and the Formation of Bipolar Planetary Nebulae
}

\author{
Vikram V. Dwarkadas \& Stanley Owocki \\ Bartol Research Institute, Univ of Delaware, 217 Sharp Lab, Newark, \\ DE 19716
}

\begin{abstract}
We study the role of radiatively driven winds in the formation of PNe. The modulation of these winds due to stellar rotation, and Von Zeipel gravity darkening, are both considered. Rotation produces a wind that is faster at the poles but denser at the equator. The Von Zeipel effect, which states that the stellar flux is proportional to the effective surface gravity, results in the inverse density distribution, giving a wind that is both faster and denser at the poles. Both these effects produce bipolar nebulae for large rotational velocities of the star.
\end{abstract}

\section{Introduction}

Planetary nebulae (PNe) exhibit a diverse morphology, as outlined so effectively in these proceedings. While there appears to be no universally accepted morphological classification (see Manchado, these proceedings), it is quite clear that the majority of PNe do not show spherical symmetry. The predominance of non-spherical planetaries has led to much speculation regarding the origins of the asphericity. One of the commonly accepted models, the Generalized Interacting Stellar Winds model (see Frank 1999 and references within), attributes the deviation from spherical structure to a higher density in the equatorial regions of the surrounding material. This inhibits the nebular expansion at the equator, leading to a prolate or bipolar morphology.

This idea has been explored by many authors (Mellema \& Frank 1995; Dwarkadas, Chevalier and Blondin 1996), who have shown that, depending on the nature of the asymmetry in the surrounding medium, a vast variety of morphological shapes can be obtained. The origin of this enhanced equatorial density distribution however remains unknown. Suggestions include the presence of a disk, which could be formed by rotation or common envelope evolution; the presence of magnetic fields, and the role of binary companions (Livio 1995). None of these models however are generally applicable, and each requires finetuning of the conditions. Furthermore, it is highly unlikely that an equatorially enhanced density distribution is present in all aspherical nebulae.

Herein we use the theory of radiatively driven winds to show that in the case of a rotating star, a wind driven by stellar radiation will be aspherical, with a higher velocity at the equator than at the poles. This asphericity arises naturally from the theory of line-driven winds, and the aspherical wind blowing into a spherical medium results in the formation of an elliptical or bipolar nebula. 


\section{Radiation Driven Winds}

For a star with luminosity $L$, the CAK, line-driven mass loss rate (Castor, Abbott \& Klein 1975) can be written in terms of the mass flux $\dot{m} \equiv \dot{M} / 4 \pi R^{2}$ at the stellar surface radius $R$, which then depends on the surface radiative flux $F=L / 4 \pi R^{2}$ and the effective surface gravity $g_{\text {eff }} \equiv\left(G M / R^{2}\right)(1-\Gamma)$ through (Owocki, Cranmer \& Gayley 1998; Dwarkadas \& Owocki 2002)

$$
\dot{m} \propto F^{1 / \alpha} g_{e f f}^{1-1 / \alpha} .
$$

where $\alpha<1$. For a rotating star, reduction by the radial component of the centrifugal acceleration yields an effective gravity that scales with co-latitude $\theta$ as

$$
g_{\text {eff }}(\theta)=g\left[1-\kappa_{e} F / g c-\Omega^{2} \sin ^{2} \theta\right],
$$

where $\Omega \equiv \omega / \omega_{c}$, with $\omega$ the star's angular rotation frequency, and $\omega_{c} \equiv \sqrt{g / R}$.

If the radiative flux $F$ is constant over the stellar surface, then the second term in equation (3) becomes $\kappa_{e} F / g c=\Gamma$, and application of equation (2) yields

$$
\frac{\dot{m}(\theta)}{\dot{m}(0)}=\left[\frac{g_{\text {eff }}(\theta)}{g_{\text {eff }}(0)}\right]^{1-1 / \alpha}=\left[1-\frac{\Omega^{2} \sin ^{2} \theta}{1-\Gamma}\right]^{1-1 / \alpha} .
$$

Since the exponent $1-1 / \alpha$ is negative, the mass flux from such a uniformly bright, rotating star increases from the pole $(\theta=0)$ to the equator $(\theta=90)$.

However, if $F(\theta) \propto g\left(1-\Omega^{2} \sin ^{2} \theta\right.$ ) (gravity darkening effect, von Zeipel, 1924) then this yields the mass flux scaling

$$
\frac{\dot{m}(\theta)}{\dot{m}(0)}=\frac{F(\theta)}{F(0)}=1-\Omega^{2} \sin ^{2} \theta
$$

Thus, when gravity darkening is taken into account, the mass flux is highest at the poles, and decreases towards the equator.

For an uniformly bright star, the latitudinal variation of terminal speed goes as:

$$
\frac{v_{\infty}(\theta)}{v_{\infty}(0)}=\left[\frac{g_{e f f}(\theta)}{g_{e f f}(0)}\right]^{1 / 2}=\left[1-\frac{\Omega^{2} \sin ^{2} \theta}{1-\Gamma}\right]^{1 / 2},
$$

where the polar speed itself scales as $v_{\infty}(0) \sim \sqrt{g R(1-\Gamma)}$.

For a gravity-darkened star, the scaling of the effective gravity with $1-\Omega^{2} \sin ^{2} \theta$ now implies

$$
\frac{v_{\infty}(\theta)}{v_{\infty}(0)}=\left[\frac{g_{e f f}(\theta)}{g_{e f f}(0)}\right]^{1 / 2}=\left[1-\Omega^{2} \sin ^{2} \theta\right]^{1 / 2}
$$

\section{Numerical Simulations}

Using the above scalings to describe the wind variation with latitude, we have carried out hydrodynamic simulations of such a wind running into either a constant density medium, or another wind. These simulations were carried out 


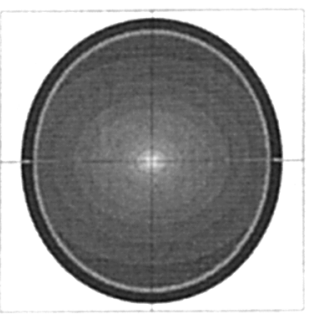

$\tilde{\Omega}=0.5$

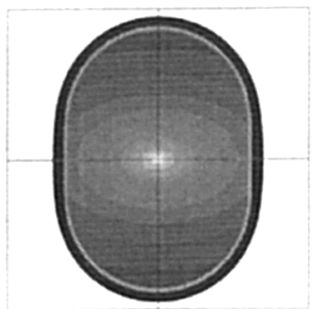

$\tilde{\Omega}=0.75$

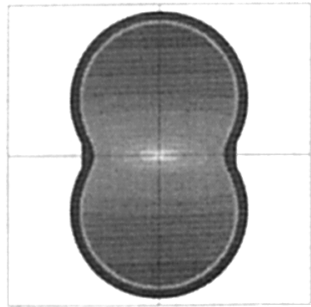

$\tilde{\Omega}=0.9$

Figure 1. Density contours from simulations of an aspherical radiatively-driven wind from a rotating star. As the rotation parameter $\tilde{\Omega}=\Omega /(1-\Gamma)$ (see text) increases, the shape of the resulting planetary nebula changes from spherical to bipolar.

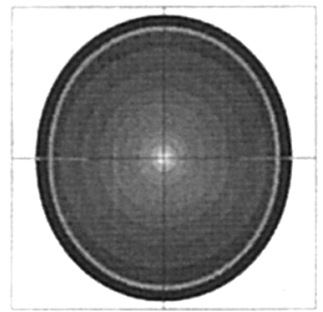

$$
\Omega=0.5
$$

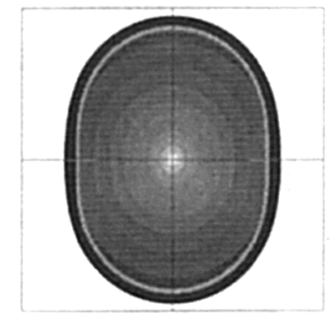

$\Omega=0.75$

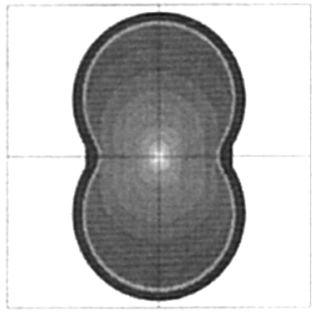

$\Omega=0.9$

Figure 2. Density contours from simulations of an aspherical radiatively-driven wind from a rotating star, when the effect of gravity darkening is taken into account. The morphology is similar to the case of pure rotation, although the interior density distribution is different.

using the VH-1 code, a 3-dimensional hydrodynamic code based on the Piecewise Parabolic Method.

Figure 1 shows results from the simulations for various values of the rotation parameter $\Omega /(1-\Gamma)$. These results are in the early stages of the expansion, when the nebula has not yet reached an energy conserving stage, and there is no hot bubble that is driving the expansion. We see that as the rotation parameter increases from about $50 \%$ of critical to about $90 \%$ of critical, the shape of the resulting nebula goes from almost spherical to distinctly bipolar.

Figure 2 shows the results of simulations when the effects of gravity darkening are also taken into account. As is seen from the figure, the overall morphology of the nebula is quite similar to the case with pure rotation, although the interior density distribution is different. 


\section{Discussion}

We have shown that radiatively driven winds from a fast rotating star can give rise to bipolar nebulae. The advantage of this model is that no external, ad-hoc asymmetry in the ambient medium is required. In the application of this theory to PN formation however, two questions arise:

- Can the winds from PNe be accurately described by the line-driven wind theory assumed herein? The mechanism that drives the wind from the central star of a PNe is not well known. However the line-driven wind mechanism is well known to explain the winds from hot stars such as $\mathrm{O}, \mathrm{B}$ and to a certain extent Wolf-Rayet stars (see Pauldrach et al., this volume). So at least for those $\mathrm{PNe}$ which have $\mathrm{O}$ and WR-type central stars, radiative driving may explain the origin of the winds. Even if the line-driven mechanism described here is not appropriate, the high stellar temperature of a PN central star makes it likely that radiation is somehow responsible for driving the wind.

The morphology of the nebula is mainly due to the asymmetric wind velocity. It is reasonable to assume that given the oblateness of a fast, rotating star, the overall trend of velocity being much higher at the poles than at the equator is quite robust. This will always result in the formation of prolate or bipolar nebula, although the exact scaling relation described herein may not apply. Such a velocity distribution is actually observed in the carbon star V Hya (Kahane et al. 1996). This leads us to the second important question:

- Are the central stars of PNe fast rotators?. PNe evolve from AGB stars, which themselves are not thought to be fast rotators. However a binary companion can spin-up the star tidally (Livio 1995). Common envelope binary mergers, where the companion spirals in and is engulfed by the primary, may lead to a spin-up during the spiraling process. For the carbon-rich star V Hya, Barnbaum et al. (1995) find that the equatorial rotation velocity may be as high as $26 \mathrm{~km} / \mathrm{s}$, a sizeable fraction of the centrifugal 'break-up' velocity. Although only a single example, it does indicate that fast rotation cannot be ruled out.

Acknowledgments. VVD is supported by NASA grant NAG5-3530, and by a grant from NASA administered by the American Astronomical Society.

\section{References}

Barnbaum, C., Morris, M., \& Kahane, C. 1995, ApJ, 450, 862

Castor, J. I., Abbott, D. C., \& Klein, R. I. 1975, ApJ, 195, 157

Dwarkadas, V. V., Chevalier, R. A., \& Blondin J. M. 1996, ApJ, 457, 773

Dwarkadas, V. V., \& Owocki, S. 2002, to be submitted

Frank, A. 1999, NewAR, 43, 31

Kahane, C, et al. 1996, A\&A, 314, 871

Livio, M. 1995, in "Asymmetrical Planetary Nebulae", eds. A. Harpaz \& N. Soker, p. 51 Mellema, G., \& Frank, A. 1995, MNRAS, 273, 401

Owocki, S. P., Cranmer, S. R., \& Gayley, K. G. 1998, ApSS, 260, 1490

von Zeipel, H. 1924, MNRAS, 84, 684 